Communications in Physics, Vol.22, No. 2 (2012), pp. 175-181

\title{
THE SIMULATION OF THE STABILIZING PROCESS OF GLASS NANOPARTICLE IN OPTICAL TWEEZER USING SERIES OF LASER PULSES
}

\author{
HO QUANG QUY \\ Academy of Military Science and Technology \\ HOANG DINH HAI \\ Pedagogical College of Nghe An, Vietnam.
}

\begin{abstract}
In this article the stable region and stabilizing process of dielectric particle in fluid by the optical tweezer using the series of laser pulses are investigated. The influence of the repetition period and number of laser pulses on the radial variance of particle and the "so-called" stable space-time pillar is simulated and discussed.
\end{abstract}

\section{INTRODUCTION}

In 1970, Ashkin [1] first demonstrated the optical trapping of particles using the radiation force produced by the focused continuous-wave (CW) beam. Since then the optical traps and tweezers have been a powerful tool for manipulating dielectric particles $[2,3]$.

Usually, the optical traps or tweezers in many experiments are conducted by using the $\mathrm{CW}$ laser. It is well known that the $\mathrm{CW}$ laser with the power of a few milliwat can only produce the radiation force with an order of a few $\mathrm{pN}$ to manipulate the microsized particles. Recently, Ambardekar et al. [4], Deng et al. [5], Zhao [6] and Wang et al. [7] used a pulsed laser to generate the large gradient force, up to $2500 \mathrm{pN}$ within a short duration, about picoseconds. Up to now, we have paid attention to optical traps using pulsed Gaussian beam (PGB) [7] and optical traps using counter-propagating pulsed Gaussian beams [8]. In works [8-11], the discussions about stability of the optical trap and the tweezers as well as the effectively controlling dielectric particles as the gold nanoparticles and live membrane, have been conducted taking into account of Brownian force. But, the stabilizing process during the pulsing of the optical beam and the absolutelystable conditions of dielectric particles were not clear. Therefore, in Ref. [12] the influence of the intensity of laser pulse and the radius of particle on the stabilizing process was simulated and discussed.

To see more clearly the stability of the particle in the optical tweezer, in this article, the influence of the total energy, beam waist, duration, repetition period and number of laser pulses on the radial variance, and dimension of the called "stable space-time pillar" are simulated and discussed. 


\section{THEORY}

A PGB is considered to trap fluctuating glass nano particles in the plate with water (Fig. 1). For simplicity, we consider the gradient optical force, which is induced by PGB acting on a Rayleigh dielectric particle. It means that the radius $a$ of the particle is much smaller than the wavelength of the laser (i.e., $a<<\lambda$ ). In this case, we can treat the dielectric particle as a point dipole. We also assume that the refractive index of the glass particle is $n_{1}$ and $n_{1}>>n_{2}$. Here $n_{2}$ is the refractive index of the water. The polarization direction of the electric field is assumed to be along the $x$ axis.

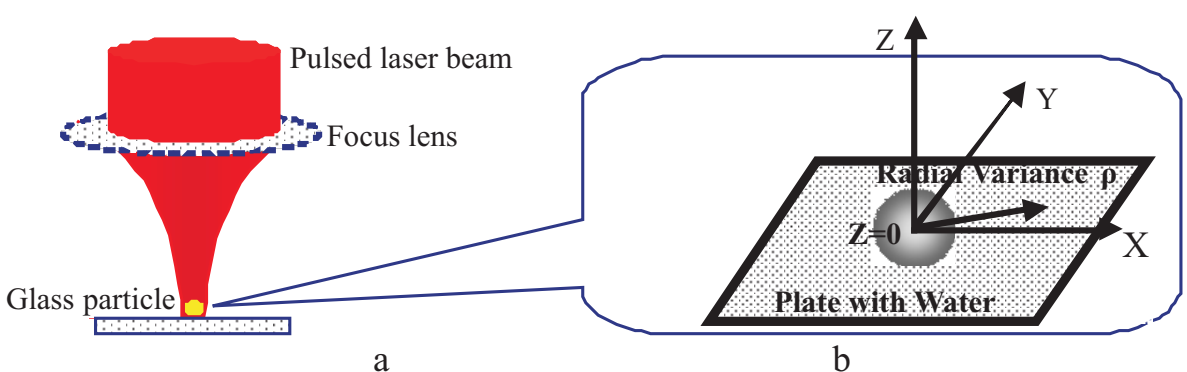

Fig. 1. (a) The schematic of optical tweezer. (b) The motion with radial variance of glass particle in the plate with water.

The expression for the electric field of the PGB is defined by [6]

$$
\begin{aligned}
& \vec{E}(\rho, z, t)=\hat{x} E_{0} \frac{i k w_{0}^{2}}{i k w_{0}^{2}+2 z} \exp \left\{-i\left[k z-\omega_{0} t\right]\right\} \\
& \times \exp \left[-i \frac{2 k z \rho^{2}}{\left(k w_{0}^{2}\right)^{2}+4 z^{2}}\right] \\
& \times \exp \left[-\frac{\left(k w_{0}^{2}\right)^{2} \rho^{2}}{\left(k w_{0}^{2}\right)^{2}+4 z^{2}}\right] \times \exp \left\{-\frac{[t-z / c]^{2}}{\tau^{2}}\right\}
\end{aligned}
$$

where $W_{0}$ is the beam waist at the plane $z=0, \rho$ is the radial coordinate, $\hat{x}$ is the unit vector of the polarization along the $x$ direction, $k=2 \pi / \lambda$ is the wave number, $\omega_{0}$ is the carrier frequency, and $\tau$ is the pulse duration. For the fixed input energy $U$ of a single pulsed beam, the constant $E_{0}$ is determined by $E_{0}^{2}=4 \sqrt{2} U /\left[n_{2} \varepsilon_{0} c W_{0}^{2}(\pi)^{3 / 2} \tau\right]$. The corresponding magnetic field under paraxial approximation can be given by

$$
\vec{H}(\rho, z, t) \cong \hat{y} n_{2} \varepsilon_{0} c \vec{E}(\rho, z, t)
$$

where $c=1 /\left(\varepsilon_{0} \mu_{0}\right)^{1 / 2}$ is the light speed in vacuum, and $\varepsilon_{0}$ and $\mu_{0}$ are the dielectric constant and permeability in the vacuum, respectively.

From the definition of the Poynting vector, we can readily obtain the intensity distribution for the PGB as follows:

$$
I(\rho, z, t)=\langle\vec{S}(\rho, z, t)\rangle=\hat{z} \frac{P}{1+4 z^{2}} \exp \left[-\frac{2 \tilde{\rho}^{2}}{1+4 \tilde{z}^{2}}\right] \exp \left[-2\left(\tilde{t}-\frac{2 \tilde{z} k w_{0}^{2}}{c \tau}\right)\right]
$$

where $P=2 \sqrt{2} U /\left[(\pi)^{3 / 2} W_{0}^{2} \tau\right], \tilde{z}=z / k W_{0}^{2} \quad \tilde{\rho}=\rho / W_{0}$ and $\tilde{t}=t / \tau$. 
By argument similar to that shown in Ref. [6] for PGB, the gradient optical force acting on dielectric particle is given by

$$
\vec{F}_{\text {grad }, \rho}(\rho, z, t)=-\frac{\hat{\rho} 2 \beta I(\rho, z, t) \tilde{\rho}}{c n_{2} \varepsilon_{0} w_{0}\left(1+4 z^{2}\right)}
$$

where $\beta=4 \pi n_{2}^{2} \varepsilon_{0} a^{3}\left[\left(m^{2}-1\right) /\left(m^{2}+2\right)\right]$ is the polarizability, and $m=n_{1} / n_{2}[6,7]$.

Assuming a low Reynold's number regime [13], the Brownian motion of the dielectric particle in the optical force field (in the optical tweezer) is described by a Langevin equations as

$$
\gamma \dot{\vec{\rho}}(t)+\vec{F}_{\text {grad }, \rho} \vec{\rho}(t)=\sqrt{2 D} \gamma \vec{h}(t)
$$

where $\vec{\rho}(t)=[x(t), y(t)]$ is the dielectric particle's position in the water plate, $\gamma=6 \pi a \eta$ is its friction coefficient, $\eta$ is the medium viscosity, $\sqrt{2 D} \gamma \vec{h}(t)=\sqrt{2 D} \gamma\left[h_{x}(t), h_{y}(t)\right]$ is a vector of independent white Gaussian random processes describing the Brownian forces, $D=k_{B} T / \gamma$ is the diffusion coefficient, $T$ is the absolute temperature, and $k_{B}$ is the Boltzmann constant.

We compute the two-dimensional (2D) motion and the radial variance (position) of a glass particle in water using the Brownian dynamic simulation method. A particle - spring model is employed to represent the glass particle, and the following equation of motion is computed for each particle:

$$
\vec{\rho}(t+\delta t)-\vec{\rho}(t)=-\frac{\vec{F}_{\text {grad }, \rho}(\vec{\rho}(t))}{\gamma} \times \vec{\rho}(t) \times \delta t+\sqrt{2 D} \times \delta t \times \vec{h}(t)
$$

where $\delta t$ is the time increment of the simulation, $\vec{h}(t)$ is a random vector whose components

are chosen from the range $[-1,1]$ in each time step. $\vec{F}_{\text {grad, }, \rho}(\vec{\rho}(t))$ in Eq. (6) describes the gradient optical force acting on the particle located at position $\rho$ at time $t$. For example, at beginning time $t=0$, the glass particle is assumed to locate at the position $\rho(t=0)=0$ (at center of tweezer), then we understand that the gradient optical force $\vec{F}_{\text {grad, } \rho}\left(W_{0}, z, 0\right)$ acts on the particle, which will be located at position $\Delta \rho$ after a time increment $\delta t$.

We interest only on the radial variance of glass particle in the pulsing time (this parameter describes the stability of particle), so the simulation will be computed from beginning moment $t=-3 \tau$ (or $t=0$ ) to ending moment $t=3 \tau$ (or $t=6 \tau$ ) of the laser pulse. In following numerical simulation we choose parameters as follows: $\lambda=1.064 \mu \mathrm{m}, \mathrm{m}=$ $n_{1} / n_{2}=1.592 / 1.332, \eta=7.797 \times 10^{-1}$ Pa.s (the small glass particle and water, for instance) [6], $W_{0}=1 \div 6 \mu \mathrm{m}, a=10 \mathrm{~nm}, \tau=1 \div 10 \mathrm{ps}$, and the input power is changed by $U=0.1 \div 0.6 \mu J[7], T=25^{\circ} \mathrm{C}$. The gradient optical force $F_{\text {grad }, \rho}$ is calculated by expression (4) in the ranges: $t=(-3 \div 3) \tau$ at $z=0 \mu m$ (consider the beam waist of pulsed Gaussian beam located in the traping plane $z=0$ ).

\section{SIMULATED RESULTS}

The radial variance of the glass particle at initial position $\rho(0)=0$ with radius of $10 \mathrm{~nm}$ as a function of pulsing time, it means as a function of pulse power or of the optical force, is shown in Fig.2a. At the begining and ending of laser pulse, when the optical force is weak, the particle moves randomly under Brownian law. With the increasing of 
the gradient optical force from zero to peak (depends on time), the glass particle is pulled into center of tweezer, where $\rho=0$, and fluctuates in the stable region, which is defined as a "called" space-time pillar with stable radius $\rho_{s t}$ and stable time $\Delta t_{s t}$ (Fig. $2 \mathrm{~b}$ ). The fluctuation of the glass particle in Fig. 2a is in a good agreement with experimental and theoretical ones for phagocytic membrane [9] and gold nanoparticles [10], respectively. The glass particle is pushed outside the stable pillar when the gradient force decreases from peak to a certain value. This means that the particle will be stable during stable time $\Delta t_{s t}$, where the optical force is larger than a certain value.

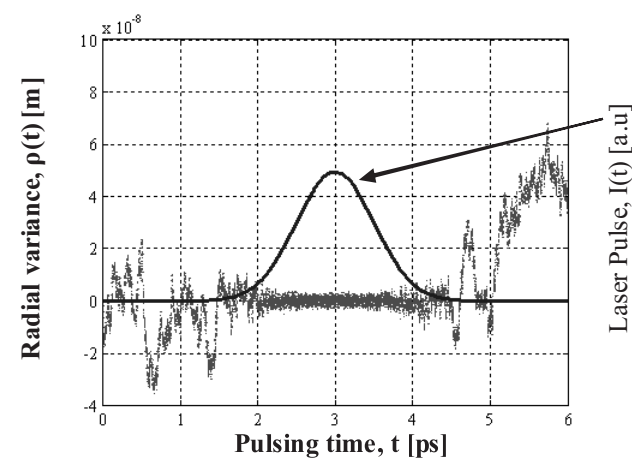

(a)

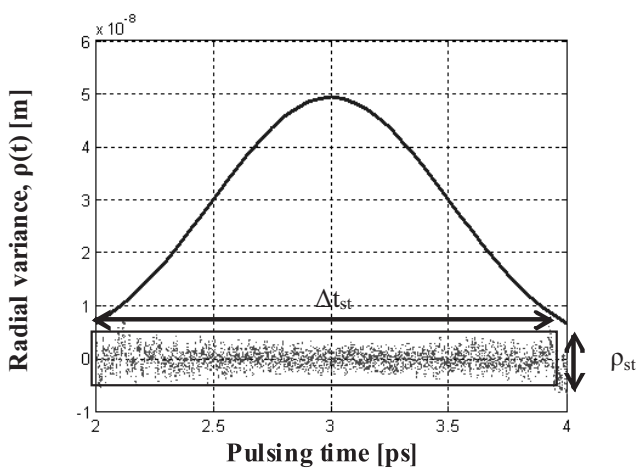

(b)

Fig. 2. (a) Radial variance of glass particle in water during pulse time. (b) The stable space-time pillar created within the interval $[-\tau, \tau]$.

From Fig. 2(b) can see that the stabilily of the glass particle depends on the dimension of this pillar $\left(\rho_{s t}\right.$ and $\left.\Delta t_{s t}\right)$, that means the stability is higher when the stable radius of pillar $\rho_{s t}$ is shorter and the stable time $\Delta t_{s t}$ is longer. Moreover, the stable radius and stable time of pillar depend on optical parameters of PGB as total energy, beam waist and duration (see figures 3, 4, and 5).

From Fig. 3 to Fig. 5, we can see that the stable radius of pillar decreases and stable time of pillar increases when the total energy increases or the beam waist decreases. It means that the stability of the glass particle will be enhanced with increasing of total energy and decreasing of beam waist. But, when the duration increases, i.e. the laser temporary power decreases, the stable radius and stable time increase together. It means that the temporal stability is higher, but the spatial stability is lower.

When the priority is given to the spatial stability, i.e. using short pulse, the temporal stability can be enhanced by using a series of short pulses. This question is concerned in Fig.6, which is simulated using a series of pulses with different repetition period $T$ and number $n$.

From Fig. 6(a) can see that after a stable time $\Delta t_{s t}=2 \tau$, the glass particle is pushed outside the stable pillar, and then pulled again to it after appearing of the next pulse. The time interval noted by $T_{B}=T-\Delta t_{s t}=6 \tau-2 \tau=4 \tau$, in which the particle oscillates under Brownian law outside the stable pillar, depends on the repetition period of pulses $(T)$. When the repetition period of pulses decreases this interval is shorter, and the 

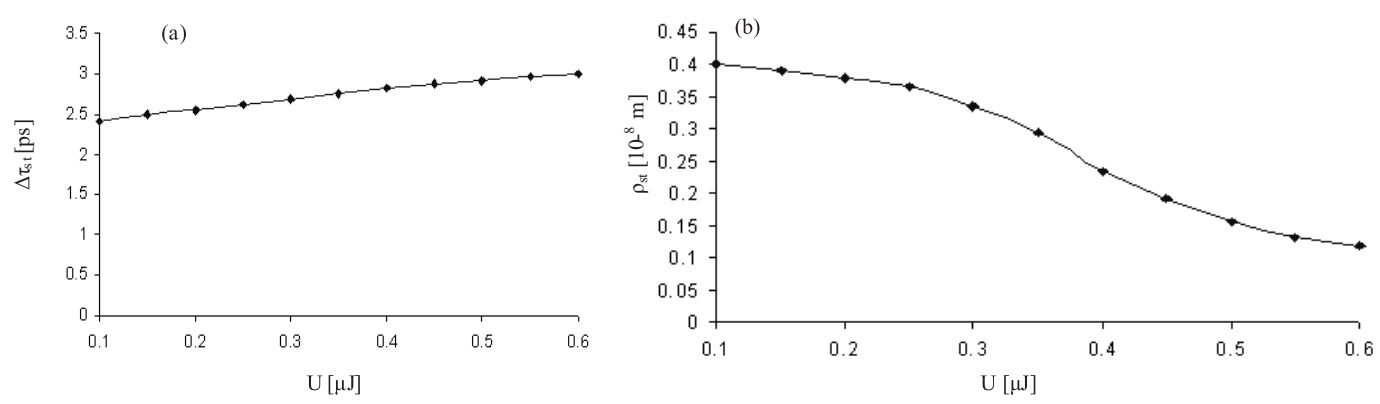

Fig. 3. a) $\Delta \tau_{s t}$ vs U. b) $\rho_{s t}$ vs U.

(a)

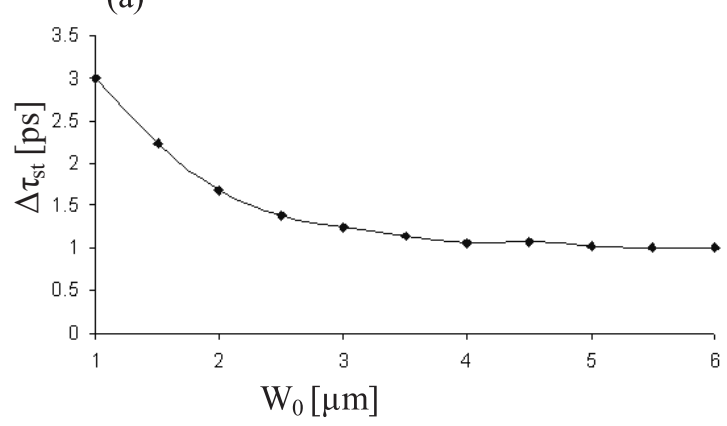

(b)

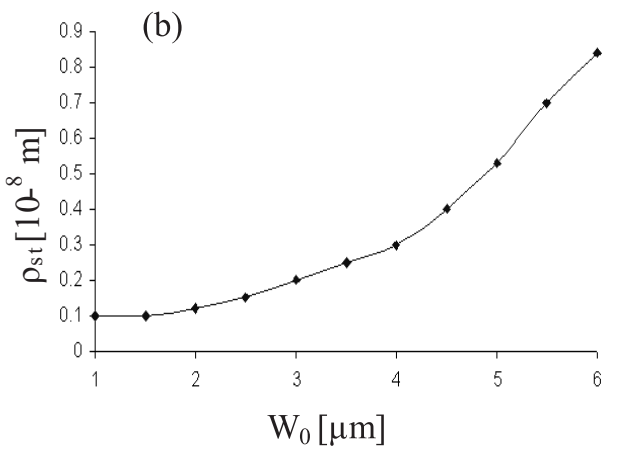

Fig. 4. a) $\Delta \tau_{s t}$ vs $\mathrm{W}_{0}$. b) $\rho_{s t}$ vs $\mathrm{W}_{0}$.
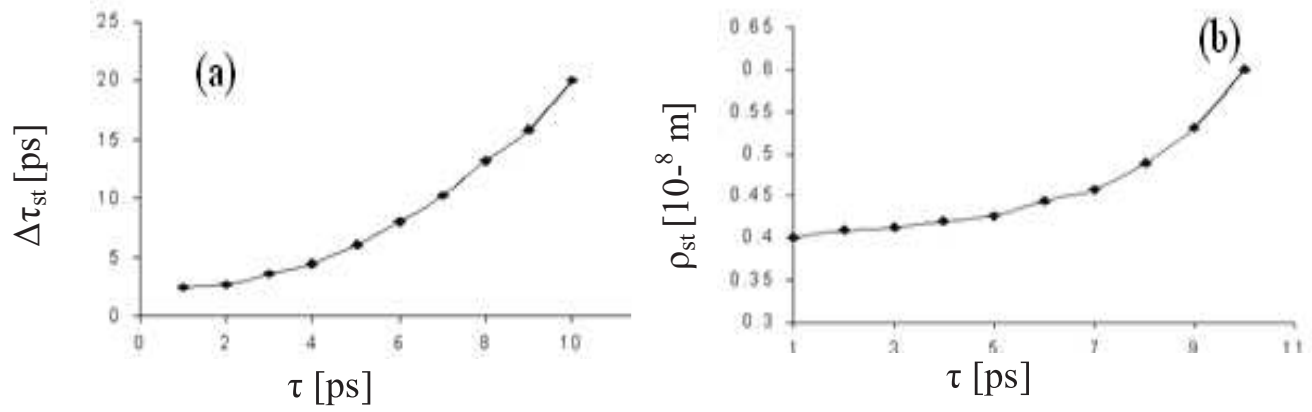

Fig. 5. $\Delta \tau_{s t}$ vs $\tau$. b) $\rho_{s t}$ vs $\tau$.

Brownian motion of the glass particle is reduced. Moreover, can see too that the spatial stability is enhanced, i.e. $\rho_{s t}$ is shorter. At least, when the repetition period is $2 \tau$ (i.e. $T_{B}=0$ in this case only) the continuous stable region appears (see Fig.6(d)), consequently, the stable time of stable pillar will be $2 n \tau$, where $n$ is the number of pulses. 


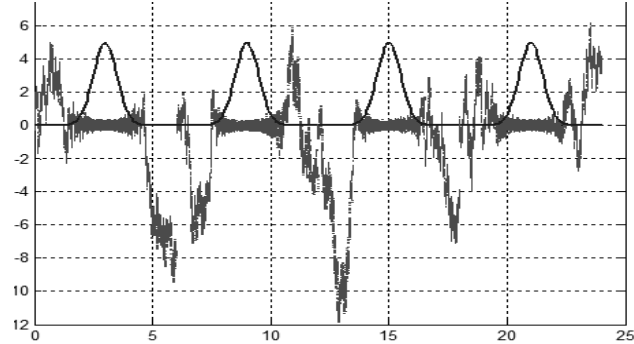

(a)

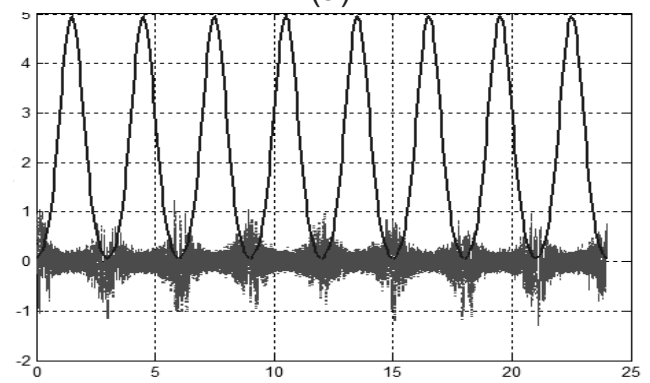

(c)

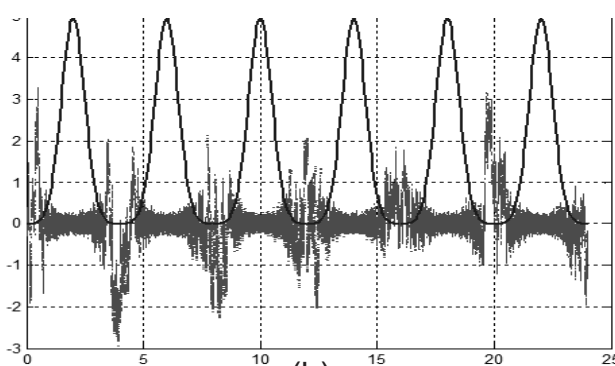

(b)

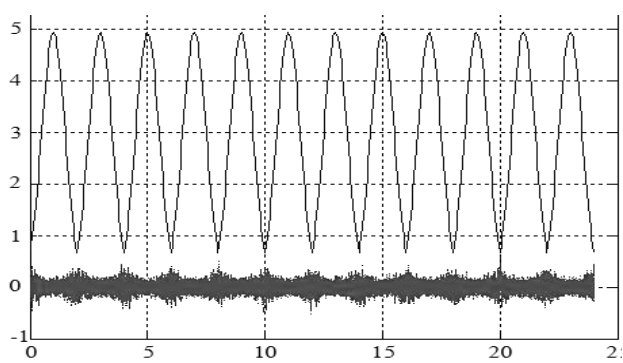

(d)

Fig. 6. Stability process of particle in optical tweezer pumped by series of laser pulses with different repetition period. (a): $T=6 \tau, n=4$; (b): $T=4 \tau, n=6$; (c): $T=3 \tau, n=8 ;(\mathrm{d}): T=2 \tau, n=12$. Note: Vertical unit [nm]; Horizontal unit $[\tau]$.

It is clear, that the stability and the dimension of the stable space-time pillar of glass nanoparticle in the optical tweezer depend not only on the total energy, beam waist of laser beam, but also on the duration, repetition period and number of the pulses.

\section{CONCLUSION}

In conclusion, we find that the Brownian force has influences on the stabilizing process of glass particles in water by the optical tweezer using PGB. The stability of glass particle in tweezer depends on the total energy, beam waist, duration of the PGB. The dimension of the stable space-time pillar can be improved by using short pulse with high repetition and large number of pulses. The simulated results can be used for other collection of principle parameters of optical tweezer.

\section{REFERENCES}

[1] A. Ashkin, "Acceleration and Trapping of Particles by Radiation Pressure," Phys. Rev. Lett. 24 (1970) 156-159.

[2] A. Ashkin, J. M. Dziedzic, J.E. Bjorkholm, S. Chu, Opt. Lett. 11 (1986) 288-290.

[3] S.C. Kuo, M. P. Sheetz, "Optical tweezers in cell biology," Trends Cell Biol. 2 (1992) 16-24.

[4] A. A. Ambardekar, Y. Q. Li, Opt. Lett. 30 (2005) 1797-1799.

[5] J. L. Deng, Q. Wei, Y. Z. Wang, Y. Q. Li, Opt. Express. 13 (2006) 3673-3680.

[6] C. L. Zhao, L. G. Wang, X. H. Lu, Phys. Let. A363 (2006) 502-506.

[7] L.G. Wang et al., Opt. Lett. 32 (2007) 1393-1395. 
[8] H. Q. Quy and M. V. Luu, Stable Manipulation Dielectric Sphere of Optical Trapping by two Counterpropagating Gaussian Pulsed Beams, IWP\&A, Nha trang, Sept.10-14 (2008) 181-186.

[9] H. Kress, Ernest H. K. Stelzer, G. Griffiths, and A. Rohrbach, Phys. Rev. E 71 (2005) 061927.

[10] Y. Seol, A. E. Carpenter, and T.T. Perkins, Opt. Lett. 31 (2006) 2429-2431.

[11] G. Volpe, G. Volpe, and D. Petrol, Phys. Rev. E76 (2007) 061118.

[12] H. Q. Quy et al., Chines Optics Letters 8 (2010) 332-334.

[13] J. Happel and H. Brenner, Low Reybnold Number Hydrodynamics, Springer, NEW York, 1983.

Received 20 June 2011. 